\title{
E-Mental Health Anwendungen für depressive und suizidale Menschen: eine Übersicht
}

\author{
Christiane Eichenberg
}

Online publiziert: 14. November 2019

(C) Der/die Autor(en) 2019

Zusammenfassung Das Internet hat sich neben verschiedenen - auch klinisch relevanten - Risiken mittlerweile als ein wichtiges Instrument in Prävention, Selbsthilfe, Beratung und sogar Therapie psychischer Erkrankungen etabliert. Eine derartige Verwendung des Internets im Kontext psychischer und psychosomatischer Erkrankungen wird unter dem Begriff E-Mental Health zusammengefasst. Online-Anwendungen gehen längst über die Informationsvermittlung durch Gesundheitswebsites hinaus und bestehen zudem aus internetbasierten Interventionsangeboten, Apps, Computerspielen, Social Media-Anwendungen oder Online-Foren. Da evidenzbasierte Therapieverfahren wie Psychotherapie und Psychopharmaka nach wie vor nicht optimal von der Mehrzahl der depressiven Patient_innen in der Bevölkerung genutzt werden, wurden mittlerweile insbesondere für Depressionen und auch Angststörungen zahlreiche Online-Anwendungen zur Behandlung entwickelt und erfolgreich evaluiert. Im Gegensatz dazu werden Online-Anwendungen für suizidale Menschen - vor allem sogenannte „Suizid-Foren“ - medial meist sehr kritisch bewertet. Im folgenden Beitrag werden daher die verschiedenen Anwendungen im E-Mental-Health Bereich für depressive und suizidale Menschen übersichtsartig vorgestellt und ihre Chancen aber auch Risiken anhand von Forschungsergebnissen kritisch eingeordnet.

Schlüsselwörter E-Mental Health · Depression · Suizidalität · Internet

C. Eichenberg $(\bowtie)$

Fakultät für Medizin, Institut für Psychosomatik, Sigmund Freud PrivatUniversität Wien, Freudplatz 3, 1020 Wien, Österreich

eichenberg@sfu.ac.at

\section{E-mental health applications for depressive and suicidal people: an overview}

Summary In addition to various risks, the Internet has meanwhile established itself as an important instrument for the prevention, self-help, counselling and even therapy and treatment of mental illnesses. Such use of the Internet in the context of mental and psychosomatic disorders is summarized under the term E-Mental Health. Online applications have long gone beyond the provision of information through health websites and also consist of Internet-based intervention services, apps, computer games, social media applications or online forums. Since evidencebased therapy methods such as psychotherapy and psychopharmaceuticals are still not optimally used by the majority of depressive patients in the population, numerous online applications for treatment of depression and anxiety disorders have now been developed and successfully evaluated. In contrast, online applications for suicidal people are usually evaluated very critically by the media, especially socalled suicide forums. In the following article, the various applications in the area of e-mental health for depressive and suicidal patients will therefore be presented in an overview and and their opportunities and risks are critically evaluated on the basis of research results.

Keywords E-Mental Health · Depression · Suicidality $\cdot$ Internet

\section{Einleitung}

Das Internet ist als modernes Informations- und Kommunikationsmedium aufgrund der Möglichkeit zur Anonymität der Nutzer_innen und inzwischen auch breiten und dauerhaften Erreichbarkeit insbe- 
sondere für psychisch kranke Menschen geeignet und vielfältig in Verwendung. Unter dem Begriff „EMental Health“ wird die Nutzung digitaler Medien in Prävention, Selbsthilfe, Beratung, Therapie und Rehabilitation psychischer und psychosomatischer Störungen verstanden, wobei mittlerweile ein breites Spektrum an Online-Anwendungen existiert: Von völlig automatisierten Selbsthilfe-Programmen, über Web- und Mobilbasierte Interventionen mit minimaler Betreuung bis hin zur klassischen therapeutischen Behandlung mittels Videokonferenz statt dem persönlichen Kontakt.

Anhand der existierenden Online-Angebote für depressive und suizidale Menschen sowie den hohen Forschungsbemühungen wird die gesamte Palette der Diskussionen um den E-Mental Health Bereich von Nutzen und Chancen, Risiken und Nebenwirkungen, Ängsten und Hoffnungen deutlich. Depressionen gehören in den Industrieländern zu den häufigsten Erkrankungen und gehen mit großem persönlichem Leidensdruck für die Betroffenen und ihre Angehörigen sowie mit einem erheblichen volkswirtschaftlichen Schaden aufgrund hoher Behandlungskosten einher (Alonso et al. 2004). Sie sind in der Regel gut behandelbar; unbehandelt führt ein chronischer Verlauf in der Regel jedoch häufig (unter anderem durch suizidale Krisen) zu einem erhöhten Mortalitätsrisiko (Blume und Hegerl 2008). In der Praxis ist schon lange eindeutig, dass bei einem überwiegenden Teil der verübten Suizide schwere depressive Syndrome eine bedeutsame Rolle spielen (Althaus und Hegel 2001). Das Wissen über die eigene Erkrankung kann Ängste und ungerechtfertigte Sorgen verringern, das Hilfesuchverhalten der Betroffenen positiv beeinflussen und die Compliance verbessern. Im Zusammenhang mit Informationsvermittlung kommt dem Internet mittlerweile eine bedeutende Rolle als wichtigstes Instrument zu, um Wissensdefizite und Vorurteile abzubauen und Hilfestellungen für depressiv Erkrankte und suizidale Menschen zu leisten. Verschiedene E-Mental Health Anwendungen für depressive Menschen sind mittlerweile gut erforscht und haben sich großteils als evident erweisen. Angebote für suizidale Menschen werden hingegen kontrovers diskutiert wobei insbesondere sogenannte Suizid-Foren eine Herausforderung darstellen. Im folgenden Beitrag werden die unterschiedlichen Anwendungsbereiche, d.h. Websites, internetbasierte Interventionsangebote, Apps, Serious Games, Social Media Networks und Online-Foren für depressive und suizidale Menschen erläutert und anhand aktueller Forschungsergebnisse diskutiert.

\section{E-Mental Health Anwendungen für depressive Menschen}

\section{Websites}

Dem Internet kommt mittlerweile eine zentrale Rolle bei der Informationsrecherche zu Gesundheitsthemen zu. Laut einer bevölkerungsrepräsentativen Studie in Deutschland (Eichenberg et al. 2013b) greifen $63,5 \%$ der deutschen Internetnutzer bei Gesundheitsfragen auf das Internet zurück. Bei psychischen Problemen würde das Internet im Bedarfsfall für ungefähr die Hälfte aller deutschen Internetnutzer eine Anlaufstelle darstellen. Im Vordergrund stünde dabei für 90,3\% aller deutschen Internetnutzer die Suche nach Informationen zu einem bestimmten gesundheitsrelevanten Thema. Der Austausch in Selbsthilfeforen $(40,8 \%)$, die Suche nach einer/einem niedergelassenen Psychotherapeut_in (30,6\%) und die Diagnostik mittels psychologischer Online-Tests (28,2\%) sind dem eindeutig nachgeordnet. Studien zeigen, dass depressive Internetnutzer_innen dieses intensiver nutzen als eine nicht-depressive Vergleichsgruppe (Katikalapudi et al. 2012), so dass davon ausgegangen werden kann, dass diese Zielgruppen gut über das Internet erreichbar ist. Nachgewiesener Maßen nutzen auch depressive Patient_innen das Internet zur Krankheitsbewältigung bzw. es gibt eine hohe Akzeptanz (z. B. Schneider et al. 2011), und dies sind inzwischen längst nicht mehr nur junge Bevölkerungsgruppen (für ältere Depressive und ihre Inanspruchnahmebereitschaft von E-Mental Health Anwendungen siehe Eichenberg et al. 2018). Umso wichtiger ist, dass die verfügbaren Angebote von hoher Qualität sind, was natürlich auch für Online-Informationen gilt, die häufig der erste Schritt in das Online-Hilfesystem sind. Eine Reihe von Studien hat daher die Qualität von Websites für unterschiedliche psychische Störungen untersucht. So kamen Grohol et al. im Jahr 2014 in ihrer Analyse von über 400 Websites zu verschiedenen psychischen Erkrankungen zu dem Ergebnis, dass die Inhaltsqualität von Seiten zur Schizophrenie, Bipolaren Störungen und Dysthymia vergleichsweise höher war als die zu Phobien und Panikstörungen (Grohol et al. 2014). Die konkreten Befunde zur Depression zeigen Folgendes: Die Websites zur Depression wurden mit der Depression-Website Content Checklist (Ferreira-Lay und Miller 2008) beurteilt. Insgesamt war die Qualität akzeptabel, allerdings zeigte sich eine große Varianz: $65 \%$ der Seiten war von schlechter Qualität. Zertifizierungen waren - wenn vorhanden - nur schwer zu finden. Öffentliche, nicht-gesponserte Informationen waren von besserer Qualität als private oder gesponserte. Griffiths et al. (2010) analysierten 21 Websites zur Depression und verglichen die Qualität der Informationen mit Depressions-Leitlinien. Ebenso untersuchten sie, ob Haftungshinweise (eindeutig Identifizierung der Autor_innen und ihre Verbindungen) auf der Seite vorhanden waren. Im 


\section{Deprexis ${ }^{\circledR}$ (www.deprexis24.de)}

Dieses kognitiv-behaviorale Programm wurde nach wissenschaftlichen Kriterien entwickelt und evaluiert (z. B. Moritz et al. 2012). Es wurde als zertifiziertes Medizinprodukt zugelassen; die Kosten werden von einzelnen deutschen Krankenkassen übernommen.

Das Programm tritt mittels dialogischem Expertensystem mit dem Nutzer in Kontakt, d.h. das Programm stellt dem Nutzenden Fragen, die dieser aus einer Auswahl vorgegebener Antworten beantwortet. Somit wird die tägliche Stimmung ermittelt, die in einer „Stimmungs-Kurve“ zur Verfügung gestellt wird. Es wird eine Psychoedukation vermittelt und Hinweise gegeben, wie mit depressiven Symptomen umzugehen ist. Außerdem werden Entspannungsübungen und kurze Hörspiele zur Verfügung gestellt.

Ergebnis konnten die Autor_innen nur einen sehr niedrigen Qualitätsscore von 4,7 auf einer Skala von 0 bis 13 konstatieren, da die Online-Informationen häufig den Empfehlungen der Leitlinie widersprechen (z. B. wurden Nebenwirkungen von Medikamenten kaum erwähnt) und sogar falsche Informationen beinhalteten. Der Haftungsscore fiel mit 5,4 (Skala 0 bis 9) etwas höher aus.

International sowie auch im deutschsprachigen Raum existieren verschiedene Ansätze, um die Qualität von gesundheitsbezogenen Internetinformationen zu sichern bzw. zu erhöhen. Die im Jahre 1995 in der Schweiz gegründete Health on the Net Foundation (www.hon.ch) hat als erste verbindliche Richtlinien zur Qualität von medizinischen Websites formuliert. Der sogenannte HONcode (HON code of conduct) ermöglicht auf der Basis acht formaler Kriterien (u.a. Sachverständigkeit, Datenschutz, Transparenz) im Sinne von Richtlinien für Webmaster mit einem Minimum an Standards die Zertifizierung von Gesundheitsportalen. Ein besonderes Angebot ist eine Suchmaschine auf der HON-Website, die ausschließlich HON-zertifizierte Informationsquellen als Suchergebnisse präsentiert. Den HONcode gibt es inzwischen in 35 Sprachen.

\section{Internetbasierte Interventionsangebote}

Es wurden zahlreiche innovative Interventionen im Bereich der psychischen Gesundheit zur Prävention und Behandlung von Depressionen entwickelt. E-Mental Health-Forschungsprojekte konzentrieren sich hauptsächlich auf die Überwachung von Symptomen (Karyotaki et al. 2015), die Früherkennung von Depressionen (Lin et al. 2013) und Sensoren und Geräte, die Vitaldaten erfassen.

Insgesamt muss bei internetbasierten Angeboten unterschieden werden, ob es sich um (therapeutenun-

\section{KEN-Online}

„Kraft der eigenen Emotionen Nutzen“ (KEN-Online) vermittelt durch verständliche Informationen und anschauliche Fallgeschichten die Grundprinzipien des Affektphobiemodells. Grundgedanke ist dabei, dass die „Gefühlsphobie“, d.h. die Angst vor den eigenen Emotionen in Kombination mit verschiedenen Abwehrmechanismen, zur Entstehung und Aufrechterhaltung von psychischen Störungen beitragen kann. Durch minimale therapeutische Unterstützung soll das transdiagnostische Programm Teilnehmer_innen, die entsprechend "gefühlsphobisch“ reagieren, dabei helfen, ihre Emotionen besser erkennen, regulieren und ausdrücken zu können.

terstütze) Selbsthilfeprogramm handelt oder um Konzepte, die auf schreibtherapeutischen Konzepten beruhen, zu denen auch die Online-Beratung zählt. Weiterhin muss differenziert werden, ob Internetangebote eine face-to-face-Therapie begleitend eingesetzt werden (sog. blended Ansätze) oder auschließlich online (zur ausführlichen Differenzierung siehe Eichenberg und Küsel 2016).

Die meisten internetbasierten Interventionsangebote sind geleitete Selbsthilfeprogramme (d.h. die Selbsthilfeaktivität wird durch - meist sehr wenige therapeutische Kontakte unterstützt) und basieren auf der kognitiven Verhaltenstherapie (CBT), daher werden sie auch als computergesteuerte kognitive Verhaltenstherapie (cCBT) bezeichnet. Die Wirksamkeit der cCBT-Therapie bei psychischen Erkrankungen ist gut dokumentiert (Berger 2015). Insbesondere cCBT für Depressionen und Angststörungen ist gut erforscht und hat sich als wirksam erwiesen (Griffiths et al. 2010). Eine Überprüfung der cCBT bei Depressionen ergab eine durchschnittliche Effektgröße (d) von 0,56 für 19 eingeschlossene randomisierte kontrollierte Studien (Eichenberg und Ott 2011). In ähnlicher Weise zeigte die Studie von Spek et al. (2007), die sich speziell mit älteren Menschen (50 Jahre und älter) befasste, dass cCBT mindestens genauso wirksam war wie eine häufig verwendete Gruppe-CBT-Intervention bei Depression. Neben den Wirksamkeitsstudien einzelner cCBT-Interventionen gibt es inzwischen mehrere internationale Metaanalysen, die hinreichend belegen, dass computergestützte kognitive Verhaltensinterventionen im Allgemeinen effektiv sind (FusterGarcia et al. 2015). In 17 der 19 untersuchten Studien wurde ein positiver Wirksamkeitsnachweis gefunden. Über alle Störungen und Interventionsformen hinweg lag der durchschnittliche Behandlungseffekt bei 0,53, was den Effektgrößen für die traditionelle ambulante Behandlung sehr ähnlich ist.

Beispiele für cCBT im Bereich der Depression sind: Help4Mood, ein agentenbasiertes Entscheidungshilfesystem für stark depressive Patient_innen (Hilvert- 
Bruce et al. 2012); THIS WAY UP, ein australisches Online-Trainingsprogramm (Vis et al. 2015); MasterMind, ein europäisches Projekt, das aus einem zehnwöchigen Trainingsprogramm auf Basis von cCBT besteht (http://seniorengage.eu), ICare, das auch präventive Ansätze verfolgt (https://www.icare-online.eu/de/ indizierte-praevention.html) oder Beating the Blues, eine englische Online-Plattform mit CBT als methodischer Basis, das Storytelling-Elemente in seine $8 \mathrm{Mo-}$ dule integriert (http://www.beatingtheblues.co.uk). Ein Beispiel aus dem deutschsprachigen Raum ist das Online-Programm Deprexis (https://www.deprexis24. de), das u.a. aus Modulen zur Verhaltensaktivierung oder kognitiven Umstrukturierung besteht (Kroenke und Spitzer 2002; siehe Kasten). Obwohl im Bereich der internetbasierten Interventionsangebote solche auf CBT-Basis dominieren, gibt es inzwischen auch gut evaluierte psychodynamische Angebote, die typischerweise weniger störungsspezifisch ausgerichtet sind. Ein Beispiel aus dem deutschsprachigen Raum ist KEN-Online (Zwerenz et al. 2017; siehe Kasten).

Ein Beispiel für ein schreibtherapeutisches Angebot ist Interapy, für das auch Evaluationen im Vergleich zur face-to-face-Therapie vorliegt (Wagner et al. 2014).

\section{Apps}

Der Einsatz von Mobilmedien im Gesundheitsbereich (sog. M-Health) wird zunehmend wichtiger, insbesondere, weil der Nutzerkreis von Handys inzwischen die Zahl der Computer- und Internetnutzer_innen um ein Vielfaches übersteigt.

Als persönliches Medium, das im Alltag ständig verfügbar ist, eignet sich das Handy ideal zur Unterstützung von Prävention, Gesundheitsförderung, Selbsthilfe und zur Unterstützung von Therapien. Schon vor 15 Jahren wurden Angebote via SMS umgesetzt, so z.B. Nachsorgeprogramme nach psychosomatischen Klinikaufenthalten (Bauer et al. 2003).

Inzwischen geht der Trend dahin, Apps anzubieten, die auf künstlicher Intelligenz basieren. Ein Beispiel hierfür ist „Woebot“ (https://woebot.io), ein Chatbot, der im Appstore heruntergeladen werden kann. Psycholog_innen der Universität Stanford haben „Woebot“ entwickelt, um Betroffenen Hilfe beim Umgang mit Depressionen und Angstzuständen zu ermöglichen - zum Beispiel für den Zeitraum, in dem sie auf einen Therapieplatz warten müssen. „Woebot“ kann aber ebenso therapiebegleitend genutzt werden. Im Kern werden kognitiv-behaviorale Techniken vermittelt, um das eigene Stimmungsmanagement $\mathrm{zu}$ verbessern. Die eigenen Stimmungen werden über die Zeit aufgezeichnet, wodurch der/dem Nutzer_in meist zuvor unbewusste Muster erkennbar werden. So lernt „Woebot“ im Laufe der Zeit seine/seinen Nutzer_in immer besser kennen, so dass die Interventionen immer passgenauer personalisiert werden können. Erste Ergebnisse sprechen für die Akzep- tanz und Wirksamkeit dieser App (Fitzpatrick et al. 2017). Wissenschaftliche Fundierung und Evaluationen entsprechender Apps sind dringend notwendig, um qualifizierte Angebote auf dem Markt anzubieten. Shen et al. (2015) konnten zeigen, dass über 1000 Apps zu Depression existieren; allerdings lagen bis 2013 lediglich 3 RCTs zu evaluierten Apps vor (Donker et al. 2013). In Zukunft gilt es, diese neuen Möglichkeiten der mobilen Selbsthilfe- und Therapieunterstützung empirisch und praktisch weiter zu fördern, $\mathrm{zu}$ evaluieren und bei positiven Evaluationsergebnissen dann in den Alltag zu integrieren.

\section{Serious Games}

Serious Games sind Computerspiele, bei denen nicht der Unterhaltungsaspekt, sondern die Lernerfahrung im Mittelpunkt steht. Sie sind nicht zur Vermittlung von Fakten gedacht, sondern dienen darüber hinaus zur praktischen Einübung von Handlungswissen und als Reflexionsmedium. Folglich liegt es auf der Hand, dass seit einigen Jahren verstärkte Bemühungen existieren Serious Games gezielt zur Gesundheitsförderung zu entwickeln, einzusetzen und zu evaluieren. Entsprechend existieren auch Serious Games zur Behandlung leichter depressiver Erkrankungen (siehe Kasten) (zur generellen Wirksamkeit von Serious Games siehe Eichenberg und Schott 2017).

Auch wenn das Forschungsfeld noch sehr jung ist, so scheint ausreichend belegt zu sein, dass Serious Games die Behandlungsmotivation besonders bei Kindern und Jugendlichen erhöhen (Brezinka 2009). Insgesamt zeigen sich sowohl Therapeut_innen wie Patient_innen offen dafür, Serious Games bei psychi-

\section{SPARX}

In dem in Neuseeland entwickelten Serious Game SPARX (www.sparx.org.nz) werden Jugendlichen, die Anzeichen einer Depression gezeigt haben Techniken aus dem Bereich der kognitiven Verhaltenstherapie vermittelt, wie zum Beispiel Umgang mit negativen Gedanken, Problemlösung, Zeiteinteilung und Entspannung. Das Spiel besteht aus sieben aufeinander aufbauenden Leveln die über einen Zeitraum von vier bis sieben Wochen ohne Therapeutenkontakt bearbeitet werden können.

Die Wirksamkeit von SPARX wurde in einer randomisierten kontrollierten Studie mit $N=187$ jungen Erwachsene untersucht. Die Proband_innen nahmen entweder an einer regulären Face-to-Face Therapie teil oder absolvierten SPARX. Am Ende der Behandlung ergab sich für die computergestützte Therapie eine mit der herkömmlichen Therapie vergleichbare Verbesserung hinsichtlich den Depressionsymptomen, der Ängstlichkeit, Hoffnungslosigkeit und der subjektiven Lebensqualität (Merry et al. 2012). 
schen Störungen zu nutzen, auch wenn der Bekanntheitsgrad noch sehr gering ist (Eichenberg et al. 2016).

Therapeutische Computerspiele haben den Vorteil, dass sie außerhalb der Therapiezeiten zeitflexibel angewendet werden und damit zwischen den Sitzungen therapeutische Erfolge stabilisieren können. Dies gilt natürlich umso mehr für Serious Games, die ebenfalls als Applikationen auf Mobilfunkgeräten verfügbar sind. Mittlerweile sind Leitlinien für die Konzipierung und Umsetzung entsprechender Spiele vorhanden (Baranowski et al. 2008).

Beispielhaft soll die Anwendungsmöglichkeit von Serious Games am Beispiel von SPARX illustriert werden, einer der wenige Serious Games, das als reine Selbsthilfeanwendung gespielt wird, d.h. nicht als Adjuvant einer Psychotherapie.

\section{E-Mental Health Anwendungen für suizidale Men- schen}

In den Medien tauchen immer mal wieder vereinzelte Berichte über Suizide auf, in denen das Internet eine Rolle spielte: entweder wurden über so genannte „Suizid-Foren" Verabredungen zu einem gemeinschaftlichen Suizid getroffen (siehe z.B. den Fall von einem jungen Norweger und der 17-jährigen Österreicherin Eva, vgl. Eichenberg 2004), Suizide in Social Media Networks angekündigt (so z.B. die 23-jährige Cynthia aus den USA, die nur wenige Stunden vor ihrem Suizid einen Abschiedsbrief auf Facebook postete, siehe http://de.ibtimes.com/articles/25231/20120125/) oder das Internet zur Beschaffung von Suizidmitteln benutzt (siehe www.spiegel.de/spiegel/print/d48902692.html). Gleichzeitig wird das Internet schon seit den 1990er Jahren von Kriseneinrichtungen als Beratungsmedium genutzt, um gezielt suizidale Menschen anzusprechen (zur Übersicht vgl. Eichenberg 2013b).

\section{Kontroverse}

Im klinischen Diskurs ist die Beurteilung der Gefahren bzw. des Nutzens von verschiedenen Internetdiensten bei suizidaler Problematik sehr heterogen. Während Websites hauptsächlich suizidpräventive Funktionen zugeschrieben werden, überwiegen bzgl. Online-Foren und sozialen Netzwerken Stimmen, die von einer immensen Schädlichkeit dieser Internetdienste ausgehen. „Werther-Effekte“ und eine Steigerung des individuellen Ausmaßes der Suizidalität werden befürchtet. Doch zu welchen Schlussfolgerungen kommt man, wenn man diese Einschätzungen mit empirischen Befunden kontrastiert? Inzwischen liegen wissenschaftlich fundierte Evidenzen für die Effekte von suizidbezogenen Websites, Online-Foren und sozialen Netzwerken vor.

\section{Websites}

Insgesamt existieren eine Reihe von inhaltsanalytischen Studien zur Qualität von Websites zu verschiedensten psychischen Störungen und Symptomen (zur Übersicht siehe Eichenberg et al. 2013a). Diese konstatieren in der Summe deutliche Qualitätsmängel (s. oben). Dieser allgemeine Befund bestätigte sich mindestens teilweise auch für Studien, die sich speziell Websites zum Problemkomplex Suizidalität widmeten. Szumilas und Kutcher (2009) analysierten beispielsweise die evidenzbasierten Inhalte bzgl. suizidaler Risikofaktoren sowie Präventionsstrategien der 20 meist besuchten Websites zum Thema Suizidalität. Lediglich $44 \%$ der Webseiten lieferten evidenzbasierte Informationen - gemeinnützige Websites dabei häufiger als privat angebotene oder gesponserte Websites.

Recupero et al. (2008) allerdings konnten in ihrer Untersuchung deutlich mehr neutrale und gegen Suizid gerichtete Websites als „Prosuizid“-Seiten identifizieren, wobei letztere zudem schwer auffindbar gewesen seien (s. auch die Hyperlink-Netzwerkanalyse von Kemp und Collings 2011). Dieses Ergebnis wird von einer weiteren Studie untermauert. Schneider et al. (2011) gingen der Frage nach, auf welche Hilfsangebote Betroffene suizidaler Krisen bei entsprechenden Internetrecherchen stoßen. Sie befragten suchtkranke Patient_innen, nach welchen Suchbegriffen sie bei Lebensüberdruss im Internet recherchieren würden; diese Schlagworte wurden jeweils in 5 Suchmaschinen eingeben und die ersten 30 Treffer klassifiziert als: Prosuizid- (PRO), Antisuizid- (ANTI), suizid-neutrale Website (NEU), Website ohne Bezug zu Suizid (NSS) oder „Error“ (ERR). Als PRO zählten alle Internetseiten, die Suizid befürworteten, dazu animierten oder Anleitungen zu suizidalem Verhalten gaben. Internetseiten wurden als neutral (NEU) eingestuft, wenn sie weder Hilfsangebote für Suizidale anboten noch zum Suizid ermutigten. Konstruktive Hilfsangebote wurden als Websites „gegen“ suizidales Verhalten (ANTI) kategorisiert. Online-Zeitungen bspw. wurden als „Nichtsuizid“-Seite (NSS) klassifiziert. Die Bezeichnung ERR erhielten Internetseiten, die nicht abrufbar waren. Als Hauptergebnis zeigte sich, dass zwar die meisten inhaltlichen Websites als ANTI-Suizid-Websites klassifiziert werden konnten (was ebenso für eine Studie an chinesischen Websites belegt wurde, siehe Chen et al. 2017), allerdings über die Hälfte der analysierten Websites keinerlei inhaltlichen Bezug zum Thema aufwiesen (d.h. als NSS klassifiziert wurden).

Dies legt insgesamt die praktische Implikation nahe, dass angesichts der anscheinenden Schwierigkeiten, auf leicht zugängliche Weise konstruktive Websites in suizidalen Krisen über das Internet $\mathrm{zu}$ recherchieren, eine Ausweitung strukturierter Hilfsangebote erfolgen muss. Denn: dass Menschen in suizidalen Krisen das Internet aufsuchen belegt auch eine aktuelle Studie (Chandler 2018). Es konnte ein leicht positiver Zusammenhang zwischen der Such- 
neigung von suizidbezogenen Begriffen bei Google und der Anzahl der Suizide nachgewiesen werden. Der Autor belegt, das dass Ausmaß dieser Beziehung von 2006 bis 2014 gewachsen sei, was auf einen zunehmende Bedeutung des Internet für Informationen zum Suizid hindeuten würde. Umso wichtiger sind konstruktive Hilfsangebote. Eine gute Anlaufstelle ist hier die Liste der Einrichtungen zur Suizidprävention (www.suizidprophylaxe.de), zusammen getragen von der Deutschen Gesellschaft für Suizidprävention (DGS), die jedoch für Betroffene noch ansprechender gestaltet werden könnte.

\section{Social Media Networks}

Die bekanntesten sozialen Netzwerkdienste in Deutschland sind z. B. Facebook, Twitter, XING oder MySpace. Insgesamt liegen nur sehr vereinzelt Studien zu suizidalen Ankündigungen von Mitgliedern auf einzelnen Social Network-Plattformen vor.

Cash (2013) bspw. analysierte suizidale Äußerungen auf MySpace. Zwar existieren keine systematischen Studien zu entsprechenden Ankündigungen z.B. auf Facebook. Allerdings scheinen die anderen User_innen in solchen Situationen mit Hilfsangeboten zu reagieren (Ruder et al. 2011), auch wenn keine Daten dazu existieren, ob entsprechend öffentliche Krisen zu Nachahmungseffekten bei anderen labilen Mitgliedern führen.

Dunlop et al. (2011) tragen mit ihrer Studie zu weiteren wichtigen Erkenntnissen bei. Sie führten Interviews mit $N=719$ Personen zwischen 14 und 24 Jahren durch, die jemanden kannten, der sich suizidierte oder es versuchte und selbst Erfahrungen mit extremer Hoffnungslosigkeit hatten. Nach einem Jahr wurde das Interview wiederholt. Als Ergebnis zeigte sich, dass $59 \%$ der Befragten zwischenzeitlich OnlineRessourcen zu Suizidalität frequentiert hatten; dazu zählten zwar auch Social Networks, deren Nutzung jedoch subjektiv keine Zunahme der eigenen Suizidalität zur Folge hatte. Dies war bei der Partizipation an Online-Foren jedoch anders: sie wurden nicht nur nach eigenen Angaben häufiger frequentiert, sondern steigerten auch den suizidalen Druck. Dieser Befund impliziert zum einen, dass auch auf diesen sog. Web 2.0-Plattformen Interventionsbedarf besteht, d.h. z. B. entsprechende Kriseneinrichtungen sich hier mit einem Verweis auf ihre Online- und Offline-Beratungsangebote deutlich sichtbar präsentieren sollten (zur Präsenz und Nutzung von psychosozialen Beratungsstellen auf Facebook siehe z. B. Lieder 2013; kritisch Thiery 2011).

Zum anderen legen die Ergebnisse von Dunlop et al. (2011) nahe, dass im Zusammenhang mit Suizidalität Foren als problematischster Online-Dienst eingeschätzt werden müssen. Dass kommunikative Online-Anwendungen differenziert betrachtet werden müssen, wird deutlich wenn wir uns die Ergebnisse des Reviews von Memon et al. (2018) speziell für jugendliche Nutzer_innen anschauen. Dieses kommt zusammenfassend $\mathrm{zu}$ dem Schluss, dass eine zeitlich intensivere Nutzung von Online-Netzwerken bei entsprechend vulnerablen Jugendlichen negative Auswirkungen haben. Dies impliziert, dass im Einzelfall erhoben werden muss, welche Angebote genau genutzt werden, d.h.: Wer nutzt in welchem Alter aus welchen Motiven und in welcher Lebenssituation welche Internetdienste?

Somit gehen auch Bestrebungen von Social Media Networks Anbietern zunehmend dahin, gefährdete Nutzer_innen automatisiert $\mathrm{zu}$ identifizieren bzw. überhaupt Nutzerdaten mit dem Ziel dahingehend auszuwerten Hochrisikopopulationen durch Data Mining und Webaktivitätsmuster zu erkennen (LopezCastroman et al. 2019). Dies ist nicht nur datenschutzrechtlich äußerst kritisch zu sehen, sondern auch aus klinisch-psychologischer Perspektive eine fragwürdige „Intervention“, denn: Es ist bekannt, dass „In-Beziehung-sein“ ein wesentlicher suizidpräventiver Faktor darstellt und für viele Menschen inzwischen auch mediale Räume zu wichtigen sozialen Räumen geworden sind. Wie können sich entsprechend vulnerable Menschen hier anderen öffnen, wenn sie befürchten müssen, dass „Maßnahmen“ eingeleitet werden aufgrund des Ausdrucks ihres psychischen Leids? Hinzu kommt, dass automatisierte Identifikationen von vermeintlich suizidalen Personen fehleranfällig sind und bei sog. „Buddy-Systemen“ (ein Nutzer kontaktiert das Netzwerk bei suizidalen Äußerungen eines anderen Nutzers) natürlich auch Missbrauch damit betrieben werden kann.

\section{Online-Foren}

So genannte „Suizidforen“ sind virtuelle Diskussionsplattformen, in denen sich vorrangig Menschen mit Suizidgedanken austauschen. Eine exakte Angabe über die Anzahl der Foren kann nicht gemacht werden, da eine hohe Fluktuation besteht. Dabei variiert das Klima der Foren und der gegenseitige Umgang mit tiefer Verzweiflung zwischen einzelnen Suizid-Portalen und hängt stark von den Motiven und der Gestaltung ihrer Forenmaster ab. Zum Beispiel können so genannte „Methodendiskussionen“ toleriert oder explizit verboten sein mit entsprechenden Sanktionen bei Nichtbeachtung. Insgesamt zählen die Suizidforen - neben z. B. Pro-Ana-Blogs - zu den so genannten „extreme communities“ (Bell 2007), d.h. Plattformen in denen sich meist Jugendliche und junge Erwachsene zu sehr prekären psychischen Problemen ohne professionelle Helfer_innen austauschen (vgl. Eichenberg 2013a).

Die empirische Forschungslage zu den Suizidforen ist zwar z.T. heterogen mit positiven wie negativen Effekten auf die Suizidneigung (für ein Review siehe Marchant et al. 2017), doch gibt es auch übereinstimmende Befunde, sogar Kulturübergreifend (z. B. Eichenberg 2008; Sueki und Eichenberg 2012; Pours- 
hirazi 2007; te Wildt und Schlimme 2008; Winkel et al. 2005). So scheint die Nutzerklientel zum einen sehr belastet und eher chronisch suizidal, zum anderen scheinen die Teilnehmer keine homogene Gruppe darzustellen, sondern die Foren mit verschiedenen (konstruktiven und destruktiven) Motiven zu nutzen, womit die Nutzung dann auch entsprechend differenzielle Effekte hat. Wichtig hierbei ist $\mathrm{zu}$ verstehen, dass Suizidforen den Mitgliedern auch Halt und soziale Unterstützung bieten können und damit präventive oder gar kurative Funktionen übernehmen. Gerade in medialen Berichten wird häufig der Eindruck erzeugt, dass solche Portale ausschließlich dazu führen, Suizidmethoden und -mittel zu verbreiten und den suizidalen Handlungsdruck zu steigern.

\section{Fazit}

E-Health, E-Mental Health, M-Health usw. werden ein immer selbstverständlicher Teil unserer Gesundheitsversorgung werden, wobei andere europäische Länder wie z. B. Schweden oder die Niederlanden im Vergleich zu den deutschsprachigen Ländern telemedizinische Dienste schon viel mehr veralltäglicht haben.

Aktuelle Trends sind z. B. Chatbots, die - auch auf Basis künstlicher Intelligenz - immer personalisierter Dialoge mit Patient_innen führen und auch Empfehlungen geben.

E-Health Anwendungen werden den Behandlungsalltag verändern. Einerseits dadurch, als dass die/der „E-informierte“ Patient_in Behandler vor neue Herausforderungen stellt (ausführlich Eichenberg und Hübner im Druck), andererseits dadurch, dass sich die therapeutische Beziehung zwischen Behandler_in und Patient_in verändert, wenn digitale Medien in Behandlungen miteinbezogen werden (z. B. ärztliche Videosprechstunden, automatisch erzeugte Daten zum Symptomverlauf via Apps, Serious Games oder internetbasierte Interventionsproramme als Adjuvant in der Therapie), ist evident (ausführlich Eichenberg und Hübner 2019). Diese Veränderungen zu reflektieren wird umso relevanter, je mehr E-Health Technologien für die Behandler_innen auch praktikabel in den Behandlungsalltag zu integrieren sind. Insgesamt stehen sowohl Behandler_innen als auch Patient_innen diesen Entwicklungen eher positiv als skeptisch gegenüber (ausführlich Eichenberg und Hübner im Druck).

Insgesamt scheint demnach eine wichtige aber auch große Aufgabe der Zukunft zu sein, die digitalen Entwicklungen im Gesundheitswesen auch von Seiten der Psychotherapeut_innen aktiv mitzugestalten, denn gerade für depressive Patient_innen gibt es nicht nur eine Vielzahl von E-Mental Health und M-Health Angeboten, sondern im Vergleich zu anderen Störungen ist die Evidenzlage hier relativ gut fundiert. Dies gilt vor allem für interbasierte Psychoedukations- und Interventionsangebote, jedoch weniger für Apps. Suizidale Patient_innen, die E-Mental Health Angebote nutzen, stellen uns jedoch vor besondere Herausforderungen. Viele Online-Angebote schließen suizidale Personen explizit daher aus, doch es fehlen Empfehlungen wie mit entsprechenden Patient_innen umgegangen werden soll, wenn sich beispielsweise erst im Verlauf der Online-Beratung eine Suizidalität manifestiert oder suizidale Absichten in Social Networks geäußert werden (für Weiterbildungen im Bereich der Online-Beratung siehe z. B. die Angebote unter. http://www.forschungsgreisslerei.at/online-2/). Die bisherigen Ansätze scheinen hier dysfunktional und in Krisen befindliche Menschen eher zu verschrecken anstatt dass sie die Vorteile - sich im Schutz der Anonymität offen äußern zu können - konstruktiv nutzen können. Denn: wenn Online-Hilfsangebote suizidale Menschen ausschließen, wird es dazu führen, dass genau diese schwere Symptomatik von den Betroffenen (weiter) verheimlich wird.

Insgesamt widersprechen die vorliegenden Ergebnisse deutlich der Annahme, das Internet stelle für suizidale Nutzer pauschal ein Gefährdungspotenzial dar. Vielmehr deuten sich für alle dargestellten Internetdienste suizidpräventive Funktionen an, die weiter ausgebaut werden sollten. So wird zukünftig für die Unterstützung von Betroffenen wichtig sein, zum einen existierende konstruktive Hilfsangebote im Internet leichter auffindbar zu machen und zum anderen auszubauen, da professionelle Beratungsangebote (siehe z.B. die Chat- und Mail-Beratung der Telefonseelsorge: http://www.telefonseelsorge.at; https://www.telefonseelsorge.de oder die Mai-Beratung des Kriseninterventionszentrum Wien: http:// www.kriseninterventionszentrum.at) nicht nur rar sind, sondern daher auch überlaufen. Dies gilt z. B. für ein vorbildliches Modellprojekt, dem sog. „[U25] Online-Beratung für junge Menschen unter 25 Jahren in Krisen und Suizidgefahr" (https://www.u25deutschland.de), das seit Jahren von Freiburg aus sehr erfolgreich mit einem Peer-Konzept arbeitet, d.h. Jugendliche von Jugendlichen beraten werden, die wiederum von hauptamtlichen Mitarbeiter_innen unterstützt und supervidiert werden. Dies ist ein gutes Beispiel dafür, wie das Internet auch für eine hochgefährdete Patientengruppe ein nutzbarer Raum in Zeiten schwerster Krisen ist.

Interessenkonflikt C. Eichenberg gibt an, dass kein Interessenkonflikt besteht.

Open Access Dieser Artikel wird unter der Creative Commons Namensnennung 4.0 International Lizenz (http:// creativecommons.org/licenses/by/4.0/deed.de) veröffentlicht, welche die Nutzung, Vervielfältigung, Bearbeitung, Verbreitung und Wiedergabe in jeglichem Medium und Format erlaubt, sofern Sie den/die ursprünglichen Autor(en) und die Quelle ordnungsgemäß nennen, einen Link zur Creative Commons Lizenz beifügen und angeben, ob Änderungen vorgenommen wurden. 


\section{Literatur}

Alonso, J.,Angermeyer, M. C., Bernert, S., etal. (2004).Disability and quality of life impact of mental disorders in europe: Results from the european study of the epidemiology of mental disorders (ESEMeD) project. Acta Psychiatr Scand, 109,38-46.

Althaus, D., \& Hegel, U. (2001). Evaluating suicide-preventive activities: the present state of research and the resulting implications. Nervenarzt, 72,677-684.

Baranowski, T., Buday, R., Thompson, D. I., \& Baranowski, J. (2008). Playing for real:video games and stories for healthrelated behavior change. American Journal of Preventive Medicine, 34(1), 74-82.

Bauer, S., Percevic, R., Okon, E., Meermann, R., \& Kordy, H. (2003). Use of text messaging in the aftercare of patients with bulimia nervosa. European Eating Disorders Review, 11(3), 279-290.

Bell, V. (2007). Online information, extreme communities and Internet therapy: Is the Internet good for our mental health? J Ment Health, 16(4), 445-457.

Berger, T. (2015). Internetbasierte Interventionen Bei Psychischen Störungen. Göttingen: Hogrefe.

Blume, A., \& Hegerl, U. (2008). Internetbasierte Kommunikation im Kompetenznetz „Depression, Suizidalität“: Erfahrungen und Chancen. In S. Bauer \& H. Kordy (Hrsg.), E-Mental-Health. Berlin, Heidelberg: Springer.

Brezinka, V. (2009). Computerspiele in der Verhaltenstherapie mit Kindern. In S. Schneider \& J. Margraf (Hrsg.), Störungen im Kindes- und Jugendalter. Lehrbuch derVerhaltenstherapie, (Bd.3, S. 234-240). Heidelberg: Springer.

Cash, S. J. (2013). Adolescent suicide statements on MySpace. Cyberpsychology, Behavior and Social Networks, 16(3), 166-174.

Chandler, V. (2018). Google and suicides: what can we learn about the use of internet to prevent suicides? Public Health, 2018(154), 144-150.

Chen, Y.Y., Hung, G.C., Cheng, Q., Tsai, C.W., \& Wu, K.C. (2017). Searching for suicide-related information on Chinese websites. Psychiatry Research, 2017(258), 506-510.

Donker, T., Petrie, K., Proudfoot, J., Clarke, J., Birch, M. R., \& Christensen, H. (2013). Smartphones for smarter delivery of mental health programs: a systematic review. J Med Internet Res, 15(11), e247.

Dunlop, S.M., et al. (2011). Where do youth learn about suicides on the Internet, and what influence does this have on suicidalideation? J of Child Psychology and Psychiatry, 52(10), 1073-1080.

Eichenberg, C. (2004). Suizidforen im Internet: Nicht generell zu verurteilen. Deutsches Ärzteblatt, 47, A-3162.

Eichenberg, C. (2008). Internet message boards for suicidal people: a typology of users. CyberPsychology \& Behavior, 11(1), 98-104.

Eichenberg, C. (2013a). Pro-Ana-Blogs, Suizid-Boards und Foren zu selbstverletzendem Verhalten. Deutsches Ärzteblatt, Ausgabe PP, 12(6), 274-276.

Eichenberg, C. (2013b). Suizidalität und Internet: Zwischen Gefährdung und Hilfe. Sozialpsychiatrische Informationen, 44(2), 17-23.

Eichenberg, C., \& Hübner, L. (2019). Digitalisierung im Gesundheitsbereich. SuchtMagazin, 45(2), 4-13.

Eichenberg, C., \& Küsel, C. (2016). Zur Wirksamkeit von Online-Beratung und Online-Psychotherapie. Resonanzen, 4,93-107.

Eichenberg, C., \&Ott, R. (2011). Klinisch-psychologischeIntervention im Internet. Psychotherapeut, 57(1), 58-69.
Eichenberg, C., \& Schott, M. (2017). Serious games for psychotherapy: a systematic review. Games for Health, 6(3), 127-135.https://doi.org/10.1089/g4h.2016.0068.

Eichenberg, C., Blokus, G., \& Malberg, D. (2013a). Evidenzbasierte Patienteninformationen im Internet: Eine Studie zur Qualität von Websites zur Posttraumatische Belastungsstörung. Zeitschrift für Psychiatrie, Psychologie und Psychotherapie, 61(4), 263-271. https://doi.org/10.1024/ 1661-4747/a000169.

Eichenberg, C., Grabmayer, G., \& Green, N. (2016). Acceptance of serious games in psychotherapy: An inquiry into the stance of therapists and patients. Telemedicine and e-health.https://doi.org/10.1089/tmj.2016.0001.

Eichenberg, C., Schott, M., Sawyer, A., Aumayr, G., \& Plößnig, M. (2018). Feasibility and conceptualization of an e-mental health treatment for depression in older adults: mixedmethods study. Journal of Medical Internet Research Aging, 1(2), e10973.https://doi.org/10.2196/10973.

Eichenberg, C., Wolters, C., \& Brähler, E. (2013b). The Internet as a mental health advisor in Germany-results of a national survey. PLoS One. https://doi.org/10.1371/journal. pone.0079206.

Eichenberg, C., Hübner, L. (in Druck) Therapeutische Beziehung im Zeitalter digitaler Medien: Perspektiven und Ergebnisse aus Verhaltenstherapie und psychodynamischer Psychotherapie.

Ferreira-Lay, P., \& Miller, S. (2008). The quality of internet information on depression for lay people. Psychiatric Bulletin, 32(5), 170-173.

Fitzpatrick, K.K., Darcy, A., \& Vierhile, M. (2017). Delivering cognitive behavior therapy to young adults with symptoms of depression and anxiety using a fully automated conversational agent (Woebot): a randomized controlled trial. JMIR Ment Health, 4(2), e19. https://doi. org/10.2196/mental.7785.

Fuster-Garcia, E., Bresó, A., Martínez-Miranda, J., Rosell-Ferrer, J., Matheson, C., \& García-Gómez, J. M. (2015). Fusing actigraphy signals for outpatient monitoring. Inf Fusion, 23, 69-80.

Griffiths, K. M., Farrer, L., \& Christensen, H. (2010). The efficacy of internet interventions for depression and anxiety disorders: a review of randomised controlled trials. Med J Aust, 192(11 Suppl), S4-S11.

Grohol, J. M., Slimowicz, J., \& Granda, R. (2014). The quality of mental health information commonly searched for on the Internet. Cyberpsychol Behav Soc Netw, 17(4), 216-221.

Hilvert-Bruce, Z., Rossouw, P.J., Wong, N., Sunderland, M., \& Andrews, G. (2012). Adherence as a determinant of effectiveness of internet cognitive behavioural therapy for anxiety and depressive disorders. Behav Res Ther, 50(7-8), 463-468.

Karyotaki, E., Kleiboer, A., Smit, F., Turner, D. T., Pastor, A. M., Andersson, G., et al. (2015). Predictors of treatment dropout in self-guided web-based interventions for depression: an 'individual patient data' meta-analysis. Psychol Med, 45(13), 2717-2726.

Katikalapudi, R., Chellappan, S., Montgomery, F., Wunsch, D., \& Lutzen, K. (2012). Associating Internet usage with depressive behavior among college students. IEEE Technology \& Society Magazine, 31(4), 73-80.

Kemp, C.G., \& Collings, S.C. (2011). Hyperlinked suicide: assessing the prominence and accessibility of suicide websites. Crisis, 32(3), 143-151.

Kroenke, K., \& Spitzer, R.L. (2002). The PHQ-9: a new depression diagnostic and severity measure. Psychiatr Ann, 32(9), 509-515. 
Lieder, S. (2013). Präsenz und Nutzung von psychosozialen Beratungsstellen auf Sozialen Netzwerken am Beispiel von Facebook. Unveröffentlichte Diplomarbeit am Department Psychologie der Universität zu Köln.

Lin, J., Ebert, D., Lehr, D., Berking, M., \& Baumeister, H. (2013). Internetbased cognitive behavioral interventions: state of the art and implementation possibilities in rehabilitation. Rehabilitation (Stuttg), 52(3), 155-163. https://doi.org/ 10.1055/s-0033-1343491.

Lopez-Castroman, J., Moulahi, B., Azé, J., Bringay, S., Guillaume, S., \& Baca-Garcia, E. (2019). Mining social networks to improve suicide prevention: A scoping review. Journal of Neuroscience Research. https://doi.org/ 10.1002/jnr.24404.

Marchant, A., Hawton, K., Stewart, A., Montgomery, P., Singaravelu, V., Lloyd, K., Ourdy, N., Daine, K., \& John, A. (2017). A systematic review of the relationship between internet use, self-harm and suicidal behaviour in young people: The good, the bad and the unknown. PLoS One, 12(8), e181722.https://doi.org/10.1371/journal.pone.0181722.

Memon, A. M., Sharma, S. G., Mohite, S. S., \& Jain, S. (2018). The role of online social networking on deliberate self-harm and suicidality in adolescents: A systematized review of literature. Indian Journal of Psychiatry, 60(4), 384-392. https://doi.org/10.4103/psychiatry.IndianJPsychiatry_ 414_17.

Merry, S. N., Stasiak, K., Shepherd, M., Frampton, C., Fleming, T., \& Lucassen, M.F. (2012). The effectiveness of SPARX, a computerised self help intervention for adolescents seeking help for depression: randomised controlled noninferiority trial. $B M J, 344$, e2598.

Moritz, S., Schilling, L., Hauschildt, M., Schröder, J., \& Treszl, A. (2012) A randomized controlled trial of internet-based therapy in depression. Behav. Res. Ther, 50, 513-521. https://doi.org/10.1016/j.brat.2012.04.006

Pourshirazi, S. (2007). „Mitreden darf nur, wer da eingetreten ist in die Finsternis“ - Über Suizidforen im Internet. Psychosozial, 30(2), 111-125.

Recupero, P.R., Harms, S.E., \& Noble, J. M. (2008). Googling suicide: surfing for suicide information on the Internet. J Clin Psychiatry, 69(6), 878-888.

Ruder, T. D., et al. (2011). Suicide announcement on Facebook. Crisis, 32(5), 280-282.

Schneider, B., Fritze, J., Georgi, K., \& Grebner, K. (2011). Finden suizidale Suchtkranke Hilfsangebote im Internet? Ner- venarzt, 82(7), 859-865. https://doi.org/10.1007/s00115010-2974-z.

Shen, N., Levitan, M. J., Johnson, A., Bender, J. L., Hamilton-Page, M., Jadad,A. R., \&Wiljer, D. (2015). Finding a depression app: a review and content analysis of the depression app marketplace. JMIR Mhealth Uhealth, 3(1), e16. https:// doi.org/10.2196/mhealth.3713.

Spek, V., Cuijpers, P., Nyklícek, I., Riper, H., Keyzer, J., \& Pop, V. (2007). Internet-based cognitive behaviour therapy for symptoms of depression and anxiety: a meta-analysis. Psychol Med,37(3),319-328.

Sueki, H., \& Eichenberg, C. (2012). Suicide bulletin board systems. Comparison between Japan and Germany. Death Studies, 36(6), 565-580.

Szumilas, M., \& Kutcher, S. (2009). Teen suicide information on the Internet: a systematic analysis of quality. La Revue canadienne de psychiatrie, 54(9), 586-604.

Te Wildt, B., \& Schlimme, J. (2008). Suizidforen im Internet als Entscheidungshilfen? Suizidprophylaxe, 35(3), 137-144.

Thiery, H. (2011). Beratung auf Facebook und Twitter. Wie Beratungsangebote auf die neuen Leitmedien reagieren können.e-beratungsjournal.net, 7(2), 1-19.

Vis, C., Kleiboer, A., Prior, R., Bønes, E., Cavallo, M., Clark, S. A., et al. (2015). Implementing and up-scaling evidencebased eMental health in Europe: the study protocol for the MasterMind project. Internet Interv, 2(4), 399-409. https://doi.org/10.1016/j.invent.2015.10.002.

Wagner, B., Horn, A.B., \& Maercker, A. (2014). Internetbased versus face-to-face cognitive-behavioral intervention for depression: a randomized controlled non-inferiority trial. J Affect Disord, 152-154, 113-121. https:// doi. org/10.1016/j.jad.2013.06.032.

Winkel, S., Groen, G., \& Petermann, F. (2005). Soziale Unterstützungin Suizidforen. Praxis der Kinderpsychologie und Kinderpsychiatrie, 54(9), 714-727.

Zwerenz, R., Becker, J., Johansson, R., Frederick, R. J., Andersson, G., \& Beutel, M.E. (2017). Psychodynamic webbased self-help intervention following inpatient psychotherapy: results of a feasibility study and randomized controlled trial. JMIR Ment Health, 4(4), e41. https:// doi. org/10.2196/mental.7889.

Hinweis des Verlags Der Verlag bleibt in Hinblick auf geografische Zuordnungen und Gebietsbezeichnungen in veröffentlichten Karten und Institutsadressen neutral. 